\title{
Improving the Reliability of Unmanned Aircraft System Wireless Communications through Cognitive Radio Technology
}

\author{
Hector Reyes*, Naima Kaabouch \\ Electrical Engineering Department, University of North Dakota, \\ Grand Forks, USA \\ Email: *hector.reyesmoncayo@my.und.edu, naima.kaabouch@engr.und.edu
}

Received May 24, 2013; revised June 24, 2013; accepted July 24, 2013

Copyright (C) 2013 Hector Reyes, Naima Kaabouch. This is an open access article distributed under the Creative Commons Attribution License, which permits unrestricted use, distribution, and reproduction in any medium, provided the original work is properly cited.

\begin{abstract}
Unmanned Aircraft System networks are a special type of networks where high speeds of the nodes, long distances and radio spectrum scarcity pose a number of challenges. In these networks, the strength of the transmitted/received signals varies due to jamming, multipath propagation, and the changing distance among nodes. High speeds cause another problem, Doppler Effect, which produces a shifting of the central frequency of the signal at the receiver. In this paper we discuss a modular system based on cognitive to enhance the reliability of UAS networks.
\end{abstract}

Keywords: UAS; Intelligent Communication Systems; Cognitive Radio; Reliability

\section{Introduction}

Wireless Communication plays a crucial role in unmanned aircraft systems (UAS) security and accomplishment of their missions [1]. UAS have recently received rapidly growing and widespread interest for military and civil purposes. The U.S. military market is projected to grow at a CAGR (Compound Annual Growth Rate) of 10\%. Revenues of $\$ 62$ billion are predicted over the period of 2010-2015 [2]. Currently, most of the applications of UAS are concentrated on the military. Their application to civil operations is still very limited due to the lack of special regulation that leverages the integration of UAS to the national airspace system (NAS) [3]. One of the concerns of the federal aviation administration (FAA) is the availability and allocation of spectrum and bandwidth for communication, command, and control so that the UAS can meet the safety regulations without conflicting with existing systems.

Unlike in conventional networks, in UAS networks there is not a dominant communication standard or technology. On the contrary, one of the problems in the development of UAS is the lack of compatibility among different platforms [4]. For example, Predator vehicles use C-band for their line of sight communications "Corresponding author. whereas Global Hawk vehicles use CDL and UHF SATCOM.

In addition to this lack of compatibility, UAS networks face other challenges including large distances and high speeds of the aircraft [5]. Large distances cause propagation losses that reduce the power and the signal to noise ratio (SNR) at the receiver, which in turn affects the availability and throughput of the data links. High speeds of aircraft produce a shifting of the frequency at the receiver called Doppler Spread. Additional problems, such as harsh environment, jamming and multipath fading as well as shadowing due to obstacles make the design of UAS networks a critical task, where it is necessary to incorporate unconventional techniques.

In this paper, we describe a methodology to increase the reliability of UAS networks. This technique is based on cognitive radio supported by software defined radio technology. Both technologies are emerging and are still in their infancy. Cognitive radio was first introduced by J. Mitola in 2000 as a solution to solve the problem of radio spectrum scarcity and its inefficient usage and allocation. Although most of the current research focuses on the scarcity of the radio spectrum problem, this technology has also the potential to enhance the reliability of dynamic networks [6] such as UAS networks. 
This paper is organized as follows. Section 2 describes the requirements and challenges in UAS networks. Section 3 gives an overview of the factors affecting the reliability of UAS networks. Finally, in Section 4 a proposed system based on cognitive radio and software defined radio is described.

\section{Requirements and Types of Failures in UAS Networks}

UAS networks need to have special attributes in order to support UAS missions. Examples of features include [7]:

- World-Wide Availability of Frequency Allocation/ Assignment-UAS should operate on frequencies that are available at all locations of interest to the user.

- Resistance to Unintentional Interference-UAS communications systems should operate successfully despite the intermittent presence of in-band signals from other RF systems.

- Resistance to Jamming-UAS have to operate successfully despite deliberate attempts to jam the wireless links

- Security: This includes privacy and resistance to deception.

In order to increase the reliability of UAS networks, the UAS communication systems need to detect failures and address them in real time. Table 1 shows a summary of the types of failures, and possible solutions. This table has been modified from [6] to fit better with UAS networks. In any case, a node failure can impact other nodes in the network. The design of reliable communication systems, as well as integration of reliable backup components, can prevent either a node failure or the spreading of a failure to the other nodes.

\section{Factors Affecting UAS Networks}

As previously mentioned, a number of factors affect the

Table 1. Types of Failures in UAS Networks.

\begin{tabular}{|c|c|}
\hline Causes of failure & Possible solutions \\
\hline Hardware failure & Health monitoring payload, backup hardware \\
\hline Propagation losses & $\begin{array}{l}\text { Spectrum sensing and channel estimation, } \\
\text { adaptive transmission, channel switching, } \\
\text { relays, mesh networks }\end{array}$ \\
\hline $\begin{array}{l}\text { Obstacles } \\
\text { and fading }\end{array}$ & $\begin{array}{l}\text { Reliable transmission, spectrum sensing and } \\
\text { channel estimation, adaptive transmission. }\end{array}$ \\
\hline Jamming & Jamming detection, channel switching \\
\hline Interference & Channel switching \\
\hline Doppler spread & Channel estimation and equalization \\
\hline Security & Jamming detection and encryption \\
\hline
\end{tabular}

reliability of UAS networks. These factors include Doppler spread, propagation losses, obstacles and fading, and interference and jamming [5]. A brief description of each of these factors is given below.

\subsection{Doppler Spread}

Due to the speed of the aircraft a phenomenon called Doppler Spread shifts the frequency at the receiver [8]. The spread of the frequency is proportional to the aircraft speed and inversely proportional to the wavelength [5] as given by:

$$
\text { Doppler spread } \propto \frac{v}{\lambda}
$$

where $v$ is the velocity and $\lambda$ is the wavelength. For example, with L-DACS1 at $600 \mathrm{~km} / \mathrm{h}$ and $1164 \mathrm{MHz}$ the Doppler spread is $1213 \mathrm{~Hz}$, while with WiMAX operating at $2.5 \mathrm{GHz}$ and vehicular speed of $100 \mathrm{Km} / \mathrm{h}$ the maximum Doppler spread is $231.5 \mathrm{~Hz}$. L-DACS1 stands for L-Band Digital Aeronautical Communication SystemType 1, a technology proposed by EUROCONTROL, the European Organization for the Safety of Air Navigation, and uses multi-carrier modulation and is based on WiMAX. In the case of L-DACS1, the Doppler spread is a significant higher portion of the OFDM subcarrier separation, $9.76 \mathrm{KHz}$, than it is in WiMAX.

Lower frequencies have longer wavelength, which in turn causes smaller Doppler spread. That means lower frequencies are better for high-speed vehicles, such as UAS. Thus, one solution to decrease the Doppler effect is to use lower frequencies for transmission; however, the radio spectrum presents more scarcity at lower frequentcies. In addition, the size of antennas in low frequencies is bigger than the size of antennas in higher frequencies.

\subsection{Propagation Losses}

UAS wireless links need to cover long distances of sometimes $360 \mathrm{~km}$, while the most common wireless link such as Wi-Fi, WiMAX, and 3GPP only cover distances of $100 \mathrm{~m}, 1 \mathrm{~km}$, and $3 \mathrm{~km}$ respectively. The strength of the signal decreases as well as the SNR, which in turn degrades the spectral efficiency that depends on the constellation size of the modulation scheme. Propagation losses are the decrease of the signal power that increases with distance and frequency. Atmospheric factors also affect the strength of the signal mainly at $\mathrm{GHz}$ frequencies. Propagation losses can increase the bit error rate (BER), decrease the SNR, produce lost packets and, thus, delay the communication, which in turn can produce a network failure. Although propagation losses are unavoidable, it is possible to reduce the number of network failures due to this phenomenon. 


\subsection{Obstacles and Fading}

Obstacles can block the signal partially or totally. Similarly, fading is the reduction of the strength of the signal at the receiver. Obstacles can reflect the signal causing multipath propagation, which in turn produces fading. Multipath propagation means that the signal takes multiple paths to travel from the transmitter to the receiver, which produces different versions of the signal in the receiver that interfere constructively or destructively depending on the difference of phase. The result of this process is the fading of the signal. In conventional design of wireless communication systems, it is a known practice to include a fading margin in the power link budget; however, in the case of mobile communications the fading effect changes; therefore, a static fading margin is not effective. Instead, an approach where the system adapts to fading changes would be more efficient. Another consequence of multipath propagation is the increase of intersymbol interference (ISI). Multipath propagation increases with motion and with the presence of man-made and natural obstacles. Precisely, UAS operate under these conditions, motion and obstacles, and thus are highly affected by this problem.

\subsection{Interference and Jamming}

Interference and jamming corrupt the signal and stop the affected nodes from transmitting signals. The term interference refers to unintended interference while jamming refers to deliberate interference. Interference becomes likely in UAS networks since the aircraft fly to different locations and can enter incumbent's coverage areas. Jamming is intentional and sometimes can be sophisticated and very aggressive. Examples of jamming techniques include constant jamming, random jamming, and deceiving jamming. Since deceiving jamming consists of misleading the legitimate nodes, this type of jamming is one of the most difficult to detect.

\section{Proposed System}

Figure 1 shows the block diagram of the proposed intelligent communication system to increase the reliability of UAS networks. This system is modular, which means the blocks can be added and removed as needed. The core of the system is the processing unit that takes the signal from the transceiver, a software defined radio (SDR), performs several processes and sends information to the decision making subsystem so that it can make decisions to control the SDR. These processes include channel estimation and spectrum sensing. Other processes include keeping the history of events in order to learn from previous experiences and speed up the processing. The processing subsystem also receives information from a GPS to be aware of the location, since the location needs to be considered in the processing and decision making.

The proposed cognitive radio (CR) system functions, cognitive cycle, can be summarized in three stages $[9,10]$ : Observing, Decision Making, and Taking Action, as shown in Figure 2. In each of these stages, there are different processes taking place that involve techniques from different fields such as digital signal processing, estimation theory, and artificial intelligence. A summary of the main aspects of each one is given below.

\subsection{Observing}

A cognitive radio must be aware of the context where it operates. This awareness includes knowledge of the environment, the communication requirements of the users, the regulatory policies and its own capabilities [10]. Knowledge of the environment mainly includes understanding the surrounding radio spectrum scene. Spectrum sensing and channel estimation support the context awareness of a CR system. Spectrum sensing is the process of obtaining awareness about the spectrum usage and existence of primary users in a determined area [11]. Channel estimation is the process of collecting the channel-state information (CSI) to assess the channel capacity [12] and its characteristics.

Sensing is critical to detect when a channel is being used by other radio systems. From the point of view of spectrum management, sensing allows for the identifica-

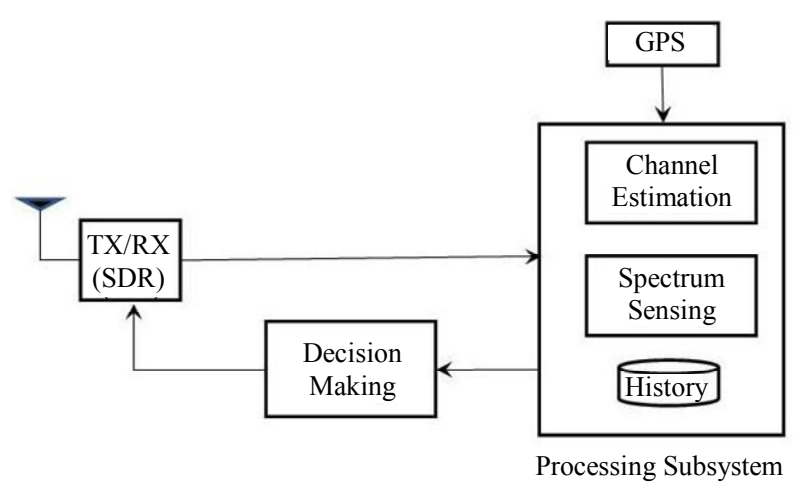

Figure 1. UAS Network Reliability System.

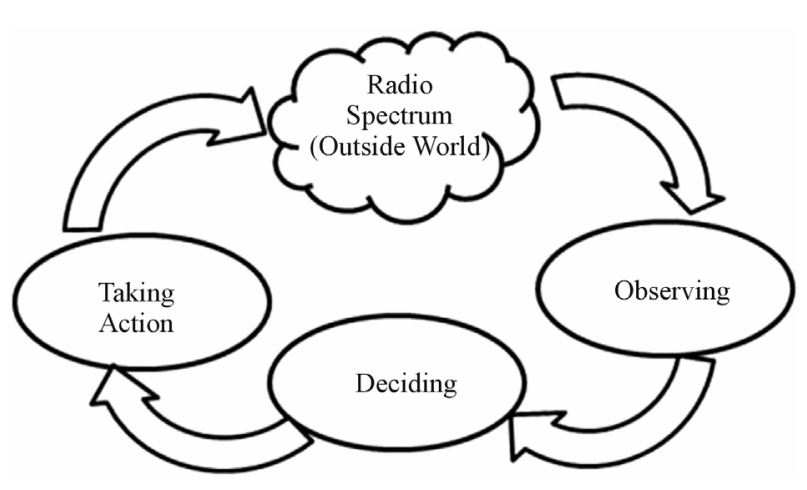

Figure 2. Cognitive Cycle. 
tion of spectrum holes to access dynamically the spectrum. From the point of view of reliability, sensing can prevent interference and jamming. SNR and RSS (received strength signal) are a basic way of sensing that help to estimate how apart the nodes are and to determine if they are about to lose connection. Knowing that allows the CR system to switch to a lower frequency channel, modify the modulation scheme, or increase the transmission power.

Channel estimation is necessary for optimal adjustment of system parameters to changing conditions. In mobile communication systems such as UAS networks the received signal strength oscillates as the vehicle travels through interference patterns caused by multipath, shadowing due to obstacles, and the change in distance between nodes. Generally, CR systems can be designed to maximize their throughput and reliability for a given quality of service (QoS). This can be accomplished by adapting the system parameters to the fluctuations created by multipath and shadowing. This process requires estimation, prediction, and tracking of the received signal as accurate as possible [13].

Channel estimation provides the CR with the information about the medium that the CR system needs to reconfigure its parameters. For example, the channel estimation subsystem can approximate the Doppler spread so that the receiver can tune itself to counteract the Doppler Effect. Equalization uses information from the channel estimation subsystem to reduce the ISI and exploit the delay diversity of the channel [14]. By using channel estimation and equalization the system can adapt to fading changes.

Estimation is a statistical process that takes samples from the physical system to calculate approximate values of the parameters of the system. Normally, the parameter is identified as $\theta$ and its estimate as $\hat{\theta}$. The main idea behind estimation is to obtain an estimate as close as possible to the actual value of the parameter. There are different approaches to deal with $\theta$ and $\hat{\theta}$. Two of the most popular estimation approaches are Bayesian estimation and Maximum likelihood estimation $[9,15]$.

The Bayesian estimation theory is based on the Bayesian risk and the costfunction of the estimation error $\epsilon=\theta-\hat{\theta}$. The goal of a Bayesian estimator is to mini-mize the Bayesian risk $R$, which is defined in (2) as the expected value $E$ of the cost function $C[16,17]$.

$$
R=E[C(\epsilon)]
$$

There are different kinds of cost functions, the most common ones are quadratic, and step function [17]. When using the quadratic cost function there is a minimum mean square error (MMSE) estimator. In the MMSE there is a need to minimize $E\left[(\theta-\hat{\theta})^{2}\right]$, where the expectation is with respect to the power density function (PDF) $p(\mathbf{x} ; \theta) . p(\mathbf{x} ; \theta)$ is the PDF of $\mathbf{x}$, the vector of input samples, with $\theta$ as a parameter. On the other hand, when using the step function, also known as "hit-or-miss" function, a maximum a posteriori (MAP) has to be estimated. The "hit-or-miss" function assigns 1 when $\epsilon>\delta$ or $\epsilon>-\delta$, where $\delta>0$ is the error threshold, and 0 otherwise. To minimize the Bayes risk with such cost function we have to maximize $p(\theta \mid \mathbf{x})$, which is the conditional, a posteriori, PDF of $\mathbf{x}$ conditioned on $\theta$ as explained in [17].

The maximum likelihood estimation (MLE) is the most popular approach to implementing practical estimators [17]. It is useful when the PDF of the parameter $\theta$ is unknown [18]. The objective of the MLE estimator is to choose $\theta$ so that the likelihood function is maximized [16-18]. Other types of estimators have also been studied and reported in the literature such as periodogram estimator, subspace estimator, blind estimator [19].

\subsection{Deciding}

The capability of making decisions is what distinguishes a cognitive radio system from a conventional radio system. This capability enables the CR system to adapt itself to fulfill the specific requirements of a determinate application. For instance, if the radio starts experiencing problems due to interference, the logical move is to switch to another channel. The CR system needs to have a strategy to know when to switch to another frequency channel, which channel is the best to switch to, etc. The goal could be maximizing throughput, reliability, or minimizing power consumption, and/or delay. All these features need to be quantifiable in order to formulate a mathematical procedure to be executed in a computer or com- putation device.

Diverse artificial intelligence techniques have been investigated and proposed by researchers to have smarter CR systems [20,21]. In [20], the authors present a survey of several artificial intelligence methods used in cognitive radio. The techniques presented are artificial neural networks (ANN), metaheuristic algorithms, hidden Markov model (HMM), rule based system (RBS), and casebased system (CBS). The literature reports the application of those techniques to different processes of CR including classification of signals for spectrum sensing $[22,23]$, radio parameter adaptation $[24,25]$, spectrum occupancy prediction $[26,27]$, and multi- objective optimization $[28,29]$.

\subsection{Taking Action}

Taking action for a $\mathrm{CR}$ is to configure its transmission and reception parameters to obtain a desired behavior in 
order to accomplish a determinate goal or set of goals [10]. The actions executed center on two main activities. The first activity is shaping the transmission profile and configuring any pertinent radio parameters to use efficiently the resources given to the $\mathrm{CR}$ and simultaneously not interfering with the resources of other radios. The second activity is reshaping the transmission profile and reconfiguring the parameters when the resources change. The resources given to a CR system are a set or frequencies, a set of time slots, and a set of antennas with beams pointed to different directions or any kind of combination of them.

\section{Conclusion}

In this paper, we have presented an overview on the main problems faced by UAS networks that can affect their communication reliability. Propagation losses due to long distances, multipath fading due to obstacles, and Doppler frequency shifting due to the high speeds of the aircraft are among these factors. To cope with those challenges, we propose an intelligent communication system based on cognitive radio that is able to adapt itself to a changing environment such as the one where UAS have to perform their missions. The proposed system is modular so that more blocks can be added to it in order to perform more complex tasks.

\section{Acknowledgments}

The authors acknowledge the support of North DakotaExperimental Program to Stimulate Competitive Research, ND EPSCoR, National Science Foundation, NSF, under grant EPS-0184442. They also acknowledge the support of Rockwell Collins under the UND-0017909 grant.

\section{REFERENCES}

[1] E. W. Frew and T. X. Brown, "Networking Issues for Small Unmanned Aircraft Systems," Journal of Intelligent and Robotic Systems, Vol. 54, No. 1-3, 2009, pp. 2137.

[2] R. K. Barnhart, "The Future of Unmanned Aircraft Systems," In: R. K. Barnhart, R. Powers and E. Shapee, Eds., Introduction to Unmanned Aircraft Systems, CRC Press, Boca Raton, 2011, p. 181.

[3] K. Dalamagkidis, K. Valavanis and L. Piegl, "On Unmanned Aircraft Systems Issues, Challenges and Operational Restrictions Preventing Integration into the National Airspace System," Progress in Aerospace Sciences, Vol. 44, No. 7, 2008, pp. 503-519.

[4] S. A. Cambone, "Unmanned Aircraft Systems Roadmap 2005-2030," 2005.

[5] R. Jain and F. Templin, "Requirements, Challenges and Analysis of Alternatives for Wireless Datalinks for Unmanned Aircraft Systems," IEEE Journal on Selected
Areas in Communications, Vol. 30, No. 5, 2012, pp. 852860.

[6] A. Azarfar, J.-F. Frigon and B. Sanso, "Improving the Reliability of Wireless Networks Using Cognitive Radios," Communications Surveys \& Tutorials, Vol. 14, No. 2, 2012, pp. 338-354.

[7] P. Fahlstrom and T. Gleason, "Introduction to UAV Systems," 2012.

[8] T. H. Eggen, J. C. Preisig and A. B. Baggeroer, "Communication over Doppler Spread Channels. II. Receiver Characterization and Practical Results," IEEE Journal of Oceanic Engineering, Vol. 26, No. 4, 2001, pp. 612-621.

[9] J. Mitola III and G. Q. Maguire Jr., "Cognitive Radio: Making Software Radios More Personal," Personal Communications, Vol. 6, No. 4, 1999, pp. 13-18.

[10] L. Doyle, "Essentials of Cognitive Radio," 2009.

[11] T. Yucek and H. Arslan, "A Survey of Spectrum Sensing Algorithms for Cognitive Radio Applications," Communications Surveys \& Tutorials, Vol. 11, No. 1, 2009, pp. 116-130.

[12] P. Demestichas, A. Katidiotis, K. A. Tsagkaris, E. F. Adamopoulou and K. P. Demestichas, "Enhancing Channel Estimation in Cognitive Radio Systems by Means of Bayesian Networks," Wireless Personal Communications, Vol. 49, No. 1, 2009, pp. 87-105. doi:10.1007/s11277-008-9559-1

[13] C. Tepedelenlioğlu, A. Abdi, G. B. Giannakis and M. Kaveh, "Estimation of Doppler Spread and Signal Strength in Mobile Communications with Applications to Handoff and Adaptive Transmission," Wireless Communications and Mobile Computing, Vol. 1, No. 2, 2001, pp. 221-242. doi: $10.1002 / \mathrm{wcm} .1$

[14] A. F. Molisch, "Wireless Communications," 2007.

[15] S. Haykin, "Cognitive Radio: Brain-Empowered Wireless Communications," IEEE Journal on Selected Areas in Communications, Vol. 23, No. 2, 2005, pp. 201-220.

[16] H. V. Poor, "An Introduction to Signal Detection and Estimation," Springer-Verlag, New York, 1988. doi:10.1007/978-1-4757-3863-6

[17] S. Kay, "Fundamentals of Statistical Signal Processing, Volume I: Estimation Theory (v. 1)" 1993.

[18] A. P. Sage and J. L. Melsa, "Estimation Theory with Applications to Communications and Control," McGrawHill, New York, 1971.

[19] E. Hossain, D. Niyato and Z. Han, "Dynamic Spectrum Access and Management in Cognitive Radio Networks," 2009.

[20] A. He, K. K. Bae, T. R. Newman, J. Gaeddert, K. Kim, R. Menon, L. Morales-Tirado, J. J. Neel, Y. Zhao and J. H. Reed, "A Survey of Artificial Intelligence for Cognitive Radios," IEEE Transactions on Vehicular Technology, Vol. 59, No. 4, 2010, pp. 1578-1592.

[21] T. W. Rondeau, "Application of Artificial Intelligence to Wireless Communications," 2007.

[22] A. F. Cattoni, M. Ottonello, M. Raffetto and C. S. Regazzoni, "Neural Networks Mode Classification Based on Frequency Distribution Features," The 2nd International Conference on Cognitive Radio Oriented Wireless Net- 
work and Communications, Orlando, 1-3 August 2007, pp. 251-257.

[23] A. Fehske, J. Gaeddert and J. Reed, "A New Approach to Signal Classification Using Spectral Correlation and Neural Networks," The First IEEE International Symposium on New Frontiers in Dynamic Spectrum Access Network, Baltimore, 8-11 November 2005, pp. 144-150.

[24] Y. Zhao, J. Gaeddert, L. Morales, K. Bae, J. S. Um and J. H. Reed, "Development of Radio Environment Map Enabled Case-and Knowledge-Based Learning Algorithms for IEEE 802.22 WRAN Cognitive Engines," The 2nd International Conference on Cognitive Radio Oriented Wireless Network and Communications, Orlando, 1-3 August 2007, pp. 44-49.

[25] Z. Zhang and X. Xie, "Intelligent Cognitive Radio: Research on Learning and Evaluation of CR Based on Neural Network," The 15th International Conference on Information and Communications Technology, Cairo, 16-18 December 2007, pp. 33-37.

[26] I. A. Akbar and W. H. Tranter, "Dynamic Spectrum Al- location in Cognitive Radio Using Hidden Markov Models: Poisson Distributed Case," Proceedings of IEEE, Richmond, 22-25 March 2007, pp. 196-201.

[27] C. H. Park, S. W. Kim, S. M. Lim and M. S. Song, "HMM Based Channel Status Predictor for Cognitive Radio," Asia-Pacific Microwave Conference, Bangkok, 11-14 December 2007, pp. 1-4.

[28] T. R. Newman, B. A. Barker, A. M. Wyglinski, A. Agah, J. B. Evans and G. J. Minden, "Cognitive Engine Implementation for Wireless Multicarrier Transceivers," Wireless Communications and Mobile Computing, Vol. 7, No. 9, 2007, pp. 1129-1142. doi:10.1002/wcm.486

[29] L. Yong, J. Hong and H. Y. Qing, "Design of Cognitive Radio Wireless Parameters Based on Multi-Objective Immune Genetic Algorithm," Vol. 1, WRI International Conference on Communications and Mobile Computing, Yunnan, 6-8 January 2009, pp. 92-96. 